\title{
OBJECTIVE SIGNS OF CONSCIOUSNESS
}

\author{
Stanislav Reinis* \\ Department of Psychology, \\ University of Waterloo, \\ Waterloo, ON, Canada \\ Received September 7, 2010; accepted October 10, 2010
}

\begin{abstract}
The human mind and consciousness under normal circumstances likely can be considered, as a unified phenomenon involving the whole brain or at least a major part of it. As suggested by some authors, the problem of consciousness cannot be solved without considering quantum mechanics. For this reason, I would like to draw on a general knowledge on quantum fields, if and how they function in the brain. What I would like to emphasize is proposal based on free electrons released during the electrophysiological events such as postsynaptic potentials, depolarization and hyperpolarization of the neuronal membrane and action potentials moving along the axons. The quantum systems form a quantum electron field in which the electrons are able to communicate one with another by means of the Pauli principle. This communication may be a carrier of the subjective consciousness which integrates and unifies functions of various parts of the brain. The output of these processes arising from the neuronal activities in various parts of the cerebral cortex and other brain areas likely may produce the content of consciousness.
\end{abstract}

Key words: Brain; Consciousness; Pauli principle; Quantum Physics

\section{INTRODUCTION}

In recent years, several papers describing various aspects of quantum human consciousness were published (Penrose, 1994, Stapp, 2007, Reinis, 2008) suggesting that now is possible to develop a reasonable theory based on quantum relations in the brain. In short, it seems acceptable that any neuronal membrane activities, pre- and postsynaptic potentials, nerve impulses and their propagation along the nerve fibers are accompanied by a generation of quantum particles, mainly electrons, that are translocated throughout the brain and elsewhere and are responsible for mutual binding of the parts of the brain into a unified functional system (binding problem), for the creation of human mind and consciousness (mind/brain problem) and for the creation of EEG (Reinis, 2008; Stapp, 2007). An important question in this respect is the subjectivity of consciousness,

\section{STATEMENT OF THE PROBLEM}

The hard problem of consciousness refers to the fact that the function of the brain considered to be a great information processor eventually may be explored and analyzed to the slightest detail, but we are still unable to explain even the most basic subjective sensations, such as those called qualia, feeling of colors, sounds and shapes.

Subjective consciousness itself is sometimes characterized as our most elementary internal experience. Everything in the universe was considered to be made up of atoms, molecules, photons, fields etc., but it seemed that the components of the mind and consciousness do not belong into any of these categories. It is believed that the consciousness is real, but utterly unexplainable in any terms familiar to science. Thus, consciousness stands as an extra fact that demands a special explanation.

To approach this problem means first to find and explore the simplest phenomena which may be involved and assembled into a fully developed human mind and consciousness. Perhaps, we might find, in these simple phenomena, the clue for the assembly of the more complex events.

Therefore, we searched for events taking place at the most basic biological and physical level, common both to the brain and to the entire universe. Such events may stand as a fundamental building block of the universe, 
and at the same time they may be able to aggregate into full-blown human consciousness.

\section{BRAIN QUANTUM FIELDS}

The human mind and consciousness are considered, under normal circumstances as a unified phenomenon involving probably the whole brain or at least a major part of it. For this reason, we have to draw on a general knowledge on quantum fields, if and how they function in the brain. As suggested by Stapp (2004, p. 250), the problem of consciousness cannot be solved without considering quantum mechanics. The question is, how to use it and what kind of dynamics is suitable for this task.

There are at least three advantages to this quantum approach: First, the temporary connection of various systems might be sufficiently fast; second, connections may be quickly initiated and terminated; and third, quantum interactions may also help to explain subjective consciousness.

From the very beginning of quantum physics, the quantum field theory has been considered a connection of quantum mechanics and special relativity (Dirac, 1925). For us, the problem is that quantum systems are incomprehensible from the viewpoint of our everyday perception and we actually do not know how they look like (Auyang, 1995).

In previously published papers several electrical phenomena in the brain generating free electrons have been proposed as a basic mechanism for the quantum field in the brain which may be formed by the electrons and quantum electron particles. They are generated continuously by all functioning neurons. The brain function is therefore represented by a state of the system of electrons (Reinis, 2008).

The field is a continuous system of interactions with infinite degrees of freedom, and also the physical effects which may be continuous. A field system is a continuous whole that can be characterized by several field variables and their interactions. It is a continuous but not amorphous entity (Auyang, 1995).

\section{PAULI EXCLUSION PRINCIPLE}

Another component of the quantum mechanics that may be useful for understanding of the brain functions is the Pauli Exclusion Principle. According to the quantum theory each electron has four quantum numbers which characterize it sufficiently. When two of the electrons differ in one number, e.g. in spin, they "communicate" this fact one to another and they may exist as closest neighbours. If they have the same spin number, they repulse each other. This is the Pauli principle and depends on Pauli force, a rather weak force of communication between the particles. This repulsive force is more than just an electrostatic repulsion between the electrons. It has a quantum mechanical character rooted in the Pauli exclusion principle, and is often called the "exclusion principle repulsion".

Although this rule is primarily valid in the atoms, it is no doubt that this is a general rule controlling the free electrons as well. This Pauli exclusive force influences only electrons close to each other. This limitation might be important.

Pauli observed that relativistic quantum field theory requires that particles with half-integer $\operatorname{spin}(s=1 / 2,3 / 2$, ...) must have antisymmetric wave functions and articles with integer $\operatorname{spin}(\mathrm{s}=0,1, \ldots)$ must have symmetric wave functions. Such observation is usually introduced as an additional postulate of quantum mechanics: The wave function of a system of electrons must be antisymmetric with respect to interchange of any two electrons. As a consequence of such principle is that two electrons with the same spin can not have the same coordinates.

The free electrons in the brain therefore communicate one with another its quantum state by means of "Pauli force", which is always repulsive and becomes very important at small distances, but is negligible at large distances. This Pauli force is quantum mechanical in origin, and keeps the charges from collapsing into a point (i.e., it keeps a negative particle and a positive particle from sitting exactly on top of one another). Some authors believe that the Pauli force has a statistical character (Dirac, 1925; Stapp, 2007).

\section{PLAYGROUND FOR MIND AND CONSCIOUSNESS}

In the brain, the particles form, all of them together, an enormously complex system of interactions which is one of the unifying principles of the brain, the human "mind". This communication is the basic fact of the Pauli principle. The electrons "feel" the presence of other electrons and respond to it. This basic function of electrons is therefore closest to the feeling of "consciousness" and seems to be meaningful to consider that this communication ability could present the most basic unit of consciousness, a basic "protoconsciousness". Intuitively, could be possible to consider whether the "spin-up" and "spin-down" units may form some kind of binary code.

To describe completely the quantum mechanical behavior of electrons in solids or semisolids, it is strictly necessary to calculate the many-electron wavefunction for the system. In principle this may be obtained from the time-independent Schroedinger equation, but in practice the potential experienced by each electron is dictated by the behavior of all the other electrons in the solid. The motion of any one electron is strongly coupled to the motion of the other electrons in the system. To solve the Schroedinger equation directly for all these electrons would thus require us to solve a system of differential equations which is beyond the capabili- 
ties of present-day computers, and is likely to remain so for the foreseeable future.

From the point of view including the quantum mechanics the model of mind and consciousness may be composed of two components. One is the mass activity of neurons, responsible for the informational content of the brain activity, and the second is the massive action of electron field that together could make the highest controlling system in the brain, the human mind, associated also with the consciousness.

\section{BOUNDARY BETWEEN PHYSICAL AND PSYCHICAL}

In this context it is possible to propose that the transition betwen physical and psychical could lie in the Pauli force interacting between electrons. Pauli force of one electron transmits very simple information only, but a mass of electrons may store a massive amount of information sufficient to construct images contained in a human mind.

The subjectivity may be given by"reading"of the quantum processes that have two faces, objective and subjectie. The objective face has the characteristes of particles as we know and study them in quantum physics. But the quantum processes has also the "subjective face" that has the characteristics which we "subjectively" perceive and know them that may determine their behavior by mechanism of observation (Stapp, 2007). Subjectivity is a function of objectively existing processes and cannot be detached from these processes.

Although we must admit that the concept such as the one offered here is purely hypothetical and may be completely wrong, it is the only one that may describe sufficiently the subjectivity of human consciousness. It was necessary to find a system where individual particles interact on a simple level. The information transferred must be simple in order to form more complex images. Therefore, the concepts such as attraction vs. repulsion, spin up and down and others have to be introduced into the model. The actual modeling of functions will be possible in the future, hopefully

When one gets to this point of reasoning, the problems of previous philosophies disappear. There is no more division into spirit and body, all the body processes move on this side of the world. There is no more idealism and its mirror image, materialism.

\section{REFERENCES}

Auyang, S. Y. (1995). How Is Quantum Field Theory Possible? New York: Oxford University Press.

Dirac, P.A.M. (1925). The Fundamental Equations of Quantum Mechanics. Proceedings of the Royal Society of London, Series A, 109, 642-653.

Pauli, W. (1981). Theory of Relativity. New York: Dover.

Penrose, R. (1994). Shadows of the Mind. Oxford: Oxford University Press.
Reinis, S. (2008). Solution of the hard problem of consciousness. Activitas Nervosa Superior, 50, 48-57.

Stapp, H. P. (2007). Mindful universe: Quantum mechanics and the participatory observer. Berlin: Springer. 